\title{
UNIFORM ASYMPTOTIC EXPANSION OF CHARLIER POLYNOMIALS
}

\author{
Bo Rui and R. Wong
}

\begin{abstract}
The Charlier polynomials $C_{n}^{(a)}(x)$ form an orthogonal system on the positive real line $x>0$ with respect to the distribution $d \alpha(x)$, where $\alpha(x)$ is a step function with jumps at the non-negative integers. Unlike classical orthogonal polynomials, they do not satisfy a second-order linear differential equation. An infinite asymptotic expansion is derived for $C_{n}^{(a)}(n \beta)$, as $n \rightarrow \infty$, which holds uniformly for $0<\varepsilon \leq \beta \leq M<\infty$. Our result includes as special cases all seven asymptotic formulas recently given by W. M. Y. Goh.
\end{abstract}

\section{Introduction}

The Charlier polynomial $C_{n}^{(a)}(x)$ can be defined by the generating function

$$
e^{-a w}(1+w)^{x}=\sum_{n=0}^{\infty} C_{n}^{(a)}(x) \frac{w^{n}}{n !}, \quad a \neq 0 .
$$

It has the explicit expression

$$
C_{n}^{(a)}(x)=\sum_{k=0}^{n}\left(\begin{array}{l}
n \\
k
\end{array}\right)\left(\begin{array}{l}
x \\
k
\end{array}\right) k !(-a)^{n-k},
$$

and satisfies the orthogonal relation

$$
\int_{0}^{\infty} C_{m}^{(a)}(x) C_{n}^{(a)}(x) d \alpha(x)=a^{n} n ! \delta_{m n}
$$

where $\alpha(x)$ is a step function with the jump

$$
d \alpha(x)=\frac{e^{-a} a^{x}}{x !}, \quad x=0,1,2, \ldots, a>0 .
$$

The three-term recurrence formula is

$$
C_{n+1}^{(a)}(x)=(x-n-a) C_{n}^{(a)}(x)-a n C_{n-1}^{(a)}(x) .
$$

For additional properties, see Chihara [2, pp. 170-172], Szegö [12, pp. 34-35], and the references cited there.

In regard to the asymptotics of the Charlier polynomials, not much is known in the literature. Unlike the classical orthogonal polynomials such as Jacobi, Laguerre,

Received June 12, 1993, revised February 23, 1994.

1991 Mathematics Subject Classification: primary 41A60, secondary 33C45.

Key words and phrases: Charlier polynomials, uniform asymptotic expansion, saddle point method, Bessel function.

The research was partially supported by the Natural Sciences and Engineering Research Council of Canada under Grant A7359. 
and Hermite, the Charlier polynomial does not satisfy a second-order linear differential equation. As a consequence, we are unable to use the vast amount of powerful asymptotic methods developed in the differential equation theory (see the definitive work by Olver [10]). Although it satisfies the second-order difference equation

$$
a \Delta^{2} C_{n}^{(a)}(x)-(x+1-a-n) \Delta C_{n}^{(a)}(x)+n C_{n}^{(a)}(x)=0,
$$

we cannot make use of it unless $x$ is a fixed number. This is mainly due to the fact that obtaining uniform asymptotic expansions of solutions of second-order linear difference equations is still not possible; see $[18,20]$. There is, of course, another possible alternative approach to this problem, that is to use its Cauchy integral representation

$$
\frac{1}{n !} C_{n}^{(a)}(x)=\frac{1}{2 \pi i} \int_{C} e^{-a w}(1+w)^{x} w^{-n-1} d w,
$$

where the contour $C$ can be a circle centered at the origin with radius less than 1 , and to apply the classical method of steepest descent (see Copson [3] or Wong [19]). But this approach also requires $x$ to be a fixed number.

In a recent paper [6], Goh has studied the asymptotic behavior of $C_{n}^{(a)}(x)$ when $x$ is a parameter depending on $n$. He divides the positive $x$-axis into seven regions:

(1) $\{x: x=\beta n, 1+\varepsilon \leq \beta \leq M\}$,

(2) $\left\{x: x=n+a+\alpha n^{1 / 2}, 2 a^{1 / 2}+\varepsilon \leq \alpha \leq M\right\}$,

(3) $\left\{x: x=n+a+2 a^{1 / 2} n^{1 / 2}+t n^{1 / 6}, t\right.$ bounded $\}$,

(4) $\left\{x: x=n+a+\alpha n^{1 / 2},-2 a^{1 / 2}+\varepsilon \leq \alpha \leq 2 a^{1 / 2}-\varepsilon\right\}$,

(5) $\left\{x: x=n+a-2 a^{1 / 2} n^{1 / 2}+t n^{1 / 6}, t\right.$ bounded $\}$,

(6) $\left\{x: x=n+a+\alpha n^{1 / 2},-M \leq \alpha \leq-2 a^{1 / 2}-\varepsilon\right\}$,

(7) $\{x: x=\beta n, \varepsilon \leq \beta \leq 1-\varepsilon\}$,

where $\varepsilon$ and $M$ are positive real numbers, and constructs an asymptotic formula for $C_{n}^{(a)}(x)$ in each of these regions. For large values of $n$, it is clear that these seven regions do not dovetail. Consequently, there are certain portions of the positive $x$-axis that are not being covered.

Goh's results remind us of the work by Tricomi [15] concerning Laguerre polynomials $L_{n}^{(\alpha)}(x)$. Tricomi set $\nu=4 n+\alpha+2$ and derived asymptotic formulas for $x$ in each of the four regions: (i) $x=O\left(\nu^{1 / 3}\right)$, (ii) $a \nu \leq x \leq b \nu$, (iii) $x-\nu=O\left(\nu^{1 / 3}\right)$, and (iv) $x \geq c \nu$, where $a, b, c$ are fixed and $0<a<b<1<c$. Tricomi's results were later considerably improved by Erdélyi [4]. More precisely, Erdélyi gave two asymptotic formulas for $L_{n}^{(\alpha)}(\nu t)$, as $n \rightarrow \infty$, where $t$ is real. One formula holds uniformly for $-\infty<t \leq a$ and the other for $b \leq t<\infty$, where $a$ and $b$ are two fixed numbers, $0<b<a<1$. These two intervals overlap and between them cover the entire $x$-axis. Erdélyi's method is based on the differential equation satisfied by Laguerre polynomials. Recently it has been shown that the same results can be obtained from their integral representations; see [5].

The Charlier polynomials are connected to Laguerre polynomials by the relation

$$
C_{n}^{(a)}(x)=n ! L_{n}^{(x-n)}(a),
$$

see [2, p. 171]. Motivated by the recent works on Laguerre polynomials [5, 11, 13], we present in this paper an asymptotic expansion for $C_{n}^{(a)}(n \beta)$, which holds uniformly for $0<\varepsilon \leq \beta \leq M<\infty$. This region covers all seven intervals considered by Goh. 
We believe that our result actually holds uniformly for $0<\varepsilon \leq \beta<\infty$, i.e., $\beta$ may be unbounded. To justify our claim, one will most likely make use of an argument similar to that given by Olde Daalhuis and Temme [9]. We will leave this problem to a future study. It is also reasonable to ask whether there exists a uniform asymptotic expansion for $C_{n}^{(a)}(n \beta)$ in the interval $-\infty<\beta \leq \delta$, where $0<\varepsilon<\delta<1$, but we again will defer this problem to another investigation. An asymptotic formula for $C_{n}^{(a)}(x)$ when $x<0$ has been obtained earlier by Maejima and Van Assche [7] using probabilistic arguments.

\section{Reduction to a canonical integral}

Returning to (1.2), we write

$$
\frac{1}{n !} C_{n}^{(a)}(n \beta)=\frac{1}{2 \pi i} \int_{C} e^{n F(w, \beta, 1 / n)} \frac{1}{w} d w
$$

where

$$
F\left(w, \beta, \frac{1}{n}\right)=\beta \log (1+w)-\log w-\frac{a}{n} w
$$

First we make the simple change of variables

$$
w=\frac{1-s}{s}
$$

so that

$$
\frac{1}{n !} C_{n}^{(a)}(n \beta)=-\frac{e^{a}}{2 \pi i} \int_{C_{s}} e^{n f(s, \beta, 1 / n)} \frac{d s}{s(1-s)}
$$

where

$$
f\left(s, \beta, \frac{1}{n}\right)=(1-\beta) \log s-\log (1-s)-\frac{(a / n)}{s}
$$

and $C_{s}$ is a circle which contains $s=1$, but not $s=0$, and is centered at a point on the positive real axis. For $\beta>0$, it can be shown that the circle $C_{s}$ can be deformed into a loop which begins and ends at $s=-\infty$ and encircles the origin in the negative sense. This can be achieved by considering the integral in (2.1) with $C_{s}$ replaced by the contour $\Gamma$ shown in Figure 1, which consists of a large circle $|s|=R$ and a loop embracing the cut along the negative $s$-axis. For $\beta>0$, the contribution from the large circle tends to zero as $R \rightarrow \infty$. Thus we obtain

$$
\frac{1}{n !} C_{n}^{(a)}(n \beta)=+\frac{e^{a}}{2 \pi i} \int_{-\infty}^{(0+)} e^{n f(s, \beta, 1 / n)} \frac{d s}{s(1-s)} .
$$

The saddle points of $f(s, \beta, 1 / n)$, i.e., the zeros of $\partial f / \partial s$, are located at $s=s_{+}$and $s=s_{-}$, where

$$
s_{ \pm}=\frac{1}{2 \beta}\left[\left(\beta-1+\frac{a}{n}\right) \pm \sqrt{\left(\beta-1+\frac{a}{n}\right)^{2}-4 \beta \frac{a}{n}}\right] .
$$

These saddle points coalesce when $\beta=\beta_{+}$and $\beta=\beta_{-}$, where

$$
\beta_{ \pm}=\left(1 \pm \sqrt{\frac{a}{n}}\right)^{2}
$$

Note that both $\beta_{+}$and $\beta_{-}$are positive. 


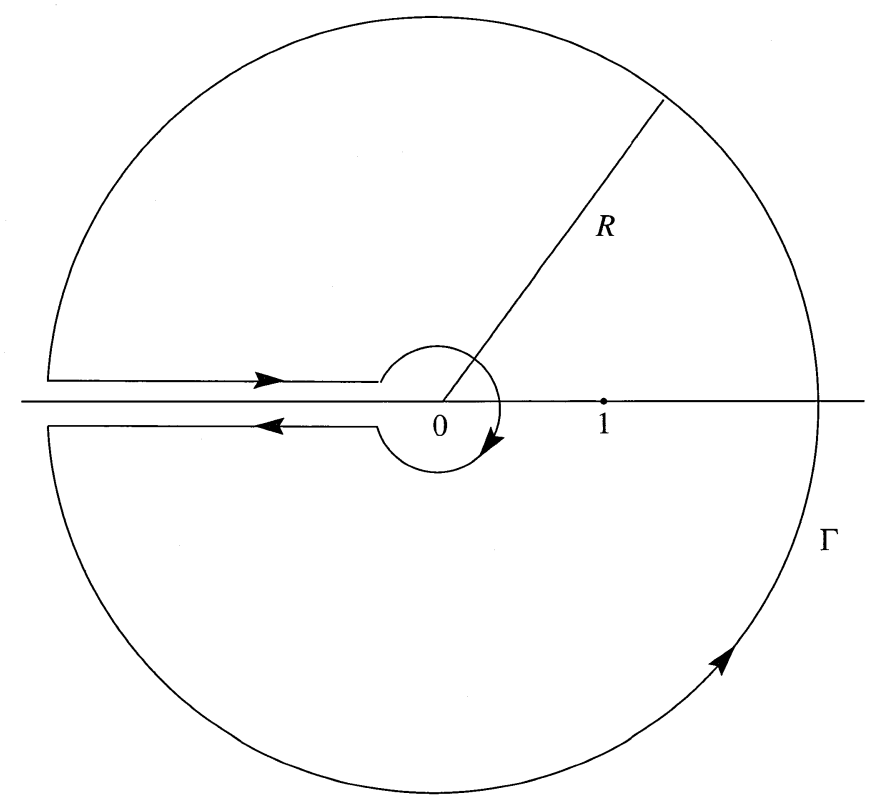

Figure 1. Contour $\Gamma$

Near $s=0$, the function $f(s, \beta, 1 / n)$ in $(2.2)$ has the expansion

$$
f\left(s, \beta, \frac{1}{n}\right)=(1-\beta) \log s+s-\frac{a / n}{s}+\frac{s^{2}}{2}+\frac{s^{3}}{3}+\cdots .
$$

Motivated by the first three terms in this expansion and by the transformations used in Temme [13] and $\mathrm{Qu}$ and Wong [11], we introduce the transformation

$$
f\left(s, \beta, \frac{1}{n}\right)=(1-\beta) \log u+u-\frac{E(\beta, n)}{u}+q(\beta, n) .
$$

For (2.6) to be analytic in the region of interest, we must have $d s / d u \neq 0$ or $\infty$. Furthermore, we require that $u=0$ corresponds to $s=0$. Now

$$
f_{s}\left(s, \beta, \frac{1}{n}\right) \frac{d s}{d u}=\frac{1-\beta}{u}+1+\frac{E(\beta, n)}{u^{2}},
$$

and $f_{s}$ vanishes at $s=s_{+}$and $s=s_{-}$. Since the right-hand side of (2.7) vanishes at $u=u_{+}$and $u=u_{-}$, where

$$
u_{ \pm}=\frac{(\beta-1) \pm \sqrt{(\beta-1)^{2}-4 E(\beta, n)}}{2},
$$

we must make $s=s_{+}$correspond to $u=u_{+}$, and $s=s_{-}$to $u=u_{-}$. This gives

$$
(1-\beta) \log s_{+}-\log \left(1-s_{+}\right)-\frac{(a / n)}{s_{+}}=(1-\beta) \log u_{+}+u_{+}-\frac{E}{u_{+}}+q
$$

and

$$
(1-\beta) \log s_{-}-\log \left(1-s_{-}\right)-\frac{(a / n)}{s_{-}}=(1-\beta) \log u_{-}+u_{-}-\frac{E}{u_{-}}+q
$$


For convenience, in (2.9) and (2.10) we have suppressed the dependence of $E$ and $q$ on $\beta$ and $n$. Since $u_{+}$and $u_{-}$are the roots of the quadratic equation $u^{2}+(1-\beta) u+E=0$, we have

$$
u_{+}+u_{-}=\beta-1 \quad \text { and } \quad u_{+} u_{-}=E
$$

from which we also obtain

$$
\frac{E}{u_{+}}+\frac{E}{u_{-}}=(\beta-1)
$$

By the same argument, we have

$$
s_{+}+s_{-}=\frac{1}{\beta}\left(\beta+\frac{a}{n}-1\right) \text { and } s_{+} s_{-}=\frac{1}{\beta} \frac{a}{n} .
$$

The last two equations together give

$$
\left(1-s_{+}\right)\left(1-s_{-}\right)=\frac{1}{\beta} \text { and } \quad \frac{a}{n}\left(\frac{1}{s_{+}}+\frac{1}{s_{-}}\right)=\beta+\frac{a}{n}-1 .
$$

Using these relations, we have upon adding and subtracting the two equations in (2.9) and $(2.10)$

$$
(1-\beta) \log E+2 q=p_{1}(\beta, n)
$$

and

$$
(1-\beta) \log \frac{(\beta-1)+\sqrt{(\beta-1)^{2}-4 E}}{(\beta-1)-\sqrt{(\beta-1)^{2}-4 E}}+2 \sqrt{(\beta-1)^{2}-4 E}=p_{2}(\beta, n),
$$

where

$$
p_{1}(\beta, n)=(1-\beta) \log \frac{a}{\beta n}+\log \beta+(1-\beta)-\frac{a}{n}
$$

and

$$
\begin{aligned}
p_{2}(\beta, n)=(1 & -\beta) \log \frac{(\beta-1+a / n)+\sqrt{(\beta-1+a / n)^{2}-4 \beta a / n}}{(\beta-1+a / n)-\sqrt{(\beta-1+a / n)^{2}-4 \beta a / n}} \\
& +\log \frac{(\beta+1-a / n)+\sqrt{(\beta-1+a / n)^{2}-4 \beta a / n}}{(\beta+1-a / n)-\sqrt{(\beta-1+a / n)^{2}-4 \beta a / n}} \\
& +\sqrt{\left(\beta-1+\frac{a}{n}\right)^{2}-4 \beta \frac{a}{n} .}
\end{aligned}
$$

The following result is useful.

Lemma. The system of nonlinear equations (2.11) and (2.12) has a unique solution $(E, q)$. For fixed $\beta \in(0, \infty)$ and $n \rightarrow \infty$, we have

$$
E(\beta, n)=\frac{a}{e} \beta^{\beta /(\beta-1)} \frac{1}{n}\left\{1-\left[\frac{\beta+1}{(1-\beta)^{2}}-\frac{2}{e(1-\beta)^{2}} \beta^{\beta /(\beta-1)}\right]\left(\frac{a}{n}\right)+o\left(\frac{1}{n}\right)\right\}
$$

if $\beta \neq 1$, and

$$
E(\beta, n)=\frac{a}{n}+o\left(\frac{a}{n}\right) \quad \text { if } \beta=1 .
$$


Also we have

$$
q(\beta, n)=(1-\beta)+\beta \log \beta+\left[\frac{\beta}{1-\beta}-\frac{1}{(1-\beta) e} \beta^{\beta /(\beta-1)}\right]\left(\frac{a}{n}\right)+o\left(\frac{1}{n}\right)
$$

if $\beta \neq 1$, and

$$
q(\beta, n)=-\frac{a}{2 n} \quad \text { if } \beta=1 .
$$

Proof. We first observe that $E$ is positive and tends to zero as $n \rightarrow \infty$, since the singular term $-E / u$ on the right-hand side of (2.6) must cancel with the singular term $-a / n s$ on the left-hand side of the equation, see the second sentence following (2.6). Hence, if we put

$$
\alpha=\frac{\sqrt{(\beta-1)^{2}-4 E}}{\beta-1},
$$

then $|\alpha|<1$. In terms of $\alpha$, equation (2.12) can be written as

$$
\log \frac{1-\alpha}{1+\alpha}=\frac{p_{2}}{\beta-1}-2 \alpha .
$$

The function on the left is monotonically decreasing in $-1<\alpha<1$ with slope $\leq-2$, and the function on the right represents a straight line with slope -2 . The graphs of these two functions clearly intersect once and only once; see Figure 2. Therefore, equation (2.20) has a unique solution $\alpha$. By (2.19), $E$ is uniquely determined, and $q$ is obtained from (2.11).

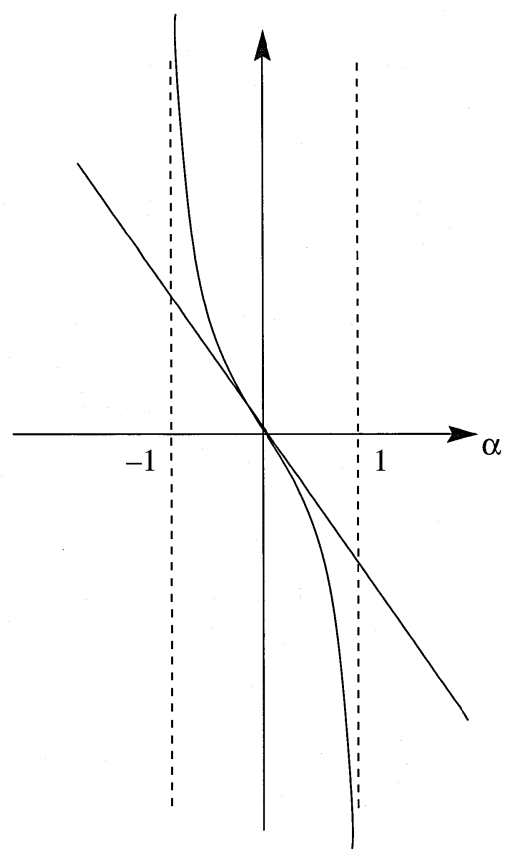

Figure 2. $\alpha$-plane 
As remarked above, $E$ tends to zero as $n \rightarrow \infty$. Hence, (2.12) can be written as

$$
\begin{aligned}
& (1-\beta) \log E-\frac{2}{1-\beta} E+o(E) \\
& \quad=(\beta-1) \log \frac{e}{a}-\beta \log \beta+(\beta-1) \log n+\frac{\beta+1}{\beta-1} \frac{a}{n}+o\left(\frac{1}{n}\right),
\end{aligned}
$$

as $n \rightarrow \infty$. Taking dominant terms on both sides, we get

$$
\log E \approx-\log n, \quad \text { as } n \rightarrow \infty .
$$

This gives the first approximation to the solution of (2.12). To improve this result, we set

$$
\log E=-\log n+\delta(\beta, n)
$$

with $\delta(\beta, n)=o(\log n)$. Inserting (2.22) into (2.21) yields

$$
(1-\beta) \delta(\beta, n)=(\beta-1) \log \frac{e}{a}-\beta \log \beta+o(1),
$$

and hence

$$
\delta(\beta, n)=\log \left[\frac{a}{e} \beta^{\beta /(\beta-1)}\right]+o(1) .
$$

Coupling (2.22) and (2.23), we obtain

$$
\log E=-\log n+\log \left[\frac{a}{e} \beta^{\beta /(\beta-1)}\right]+o(1) .
$$

To improve this approximation further, we write

$$
\log E=-\log n+\log \left[\frac{a}{e} \beta^{\beta /(\beta-1)}\right]+\varepsilon(\beta, n)
$$

and substitute it into (2.21). This leads to

$$
\varepsilon(\beta, n)=-\frac{\beta+1}{(1-\beta)^{2}} \frac{a}{n}+\frac{2}{(1-\beta)^{2}}\left(\frac{1}{e} \beta^{\beta /(\beta-1)}\right) \frac{a}{n}+o\left(\frac{1}{n}\right) .
$$

The desired result (2.15) now follows from (2.24) and (2.25).

Equation (2.16) is obtained in a similar manner. The results in (2.17) and (2.18) are obtained by substituting (2.15) and (2.16), respectively, into (2.11).

We remark that by continuing this process, higher-order terms in the asymptotic expansion of $E(\beta, 1 / n)$ can also be obtained.

In $\S 3$, it will be shown that the transformation (2.6) is one-to-one and analytic along the whole infinite loop path of integration in (2.3), and that the shape of the loop will be preserved under this transformation. Thus, changing to the variable $u$, we have

$$
\frac{1}{n !} C_{n}^{(a)}(n \beta)=\frac{e^{a+n q}}{2 \pi i} \int_{-\infty}^{(0+)} u^{-n(\beta-1)-1} \exp \left\{n\left(u-\frac{E}{u}\right)\right\} h(u) d u
$$

where

$$
h(u)=\frac{u}{(1-s) s} \frac{d s}{d u}=\frac{s}{u} \cdot \frac{\left(u-u_{+}\right)\left(u-u_{-}\right)}{\beta\left(s-s_{+}\right)\left(s-s_{-}\right)} .
$$


The second equality in (2.27) is obtained from (2.7). Note that the function $h(u)$ in (2.27) depends also on the parameters $n$ and $\beta$; thus $h(u)=h(u, \beta, n)$. However, for simplicity, we shall not indicate the dependence explicitly.

\section{The transformation (2.6)}

The properties of the mapping between $s$ and $u$ are best seen by introducing an intermediate variable $Z$ defined by

$$
(1-\beta) \log s-\log (1-s)-\frac{(a / n)}{s}=Z=(1-\beta) \log u+u-\frac{E}{u}+q .
$$

We first restrict ourselves to the case $\beta>1$. The upper half $s$-plane is shown in Figure 3. To make the function on the left-hand side of (3.1) single-valued, we introduce two cuts in the $s$-plane, one extending from $s=0$ to $s=-\infty$ along the negative real axis, and the other extending from $s=1$ to $s=+\infty$ along the positive real axis.

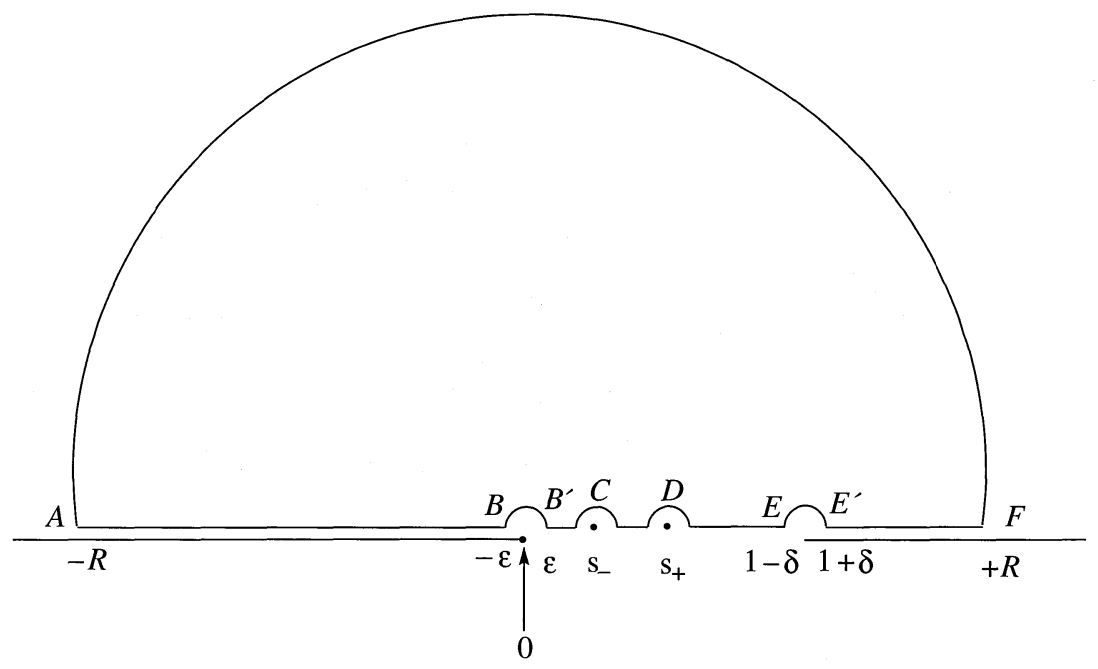

FiguRE 3. $s$-plane $(\beta>1)$

In polar coordinates, we write $s=r e^{i \theta}$, where $-\pi<\theta<\pi$. Since we are concerned only with the upper half plane, $\theta$ is restricted to $0<\theta<\pi$. Note that in this case, we have $-s=r e^{i(\theta-\pi)}$. Hence, along the top of the cut from $s=1$ to $s=+\infty$, $1-s=(s-1) e^{-i \pi}$. For example, consider the point $E^{\prime}$ located at $s=1+\delta$ in Figure 3. Since $1-s=\delta e^{-i \pi}$ and $\log (1-s)=\log \delta-i \pi$, the image of $E^{\prime}$ in the $Z$-plane is given approximately by $-\log \delta+i \pi$. In a similar manner, one can find the points $A, B, B^{\prime}, \ldots, E$ and $F$ in the complex $Z$-plane. (Some of the lines in Figure 4 are only asymptotically straight as $n \rightarrow \infty$ and as $\varepsilon, \delta \rightarrow 0$.) Note that as $s$ traverses once along the indented boundary $A B B^{\prime} C D E E^{\prime} F A$ in Figure 3, $Z$ also traverses exactly once along the corresponding curve in Figure 4. We treat the straight lines $B^{\prime} C, C D$ and $D E$ in Figure 4 as distinct parts of the boundary (see [16, p. 375, lines 22-25]). Hence, by Theorem 4.5 in [8, vol. 2, p. 118], $f(s)=(1-\beta) \log s-\log (1-s)-a / n s$ is one-to-one in the interior of the region bounded by this curve (see also $[14, \S \S 6.45$ and 6.46]). 


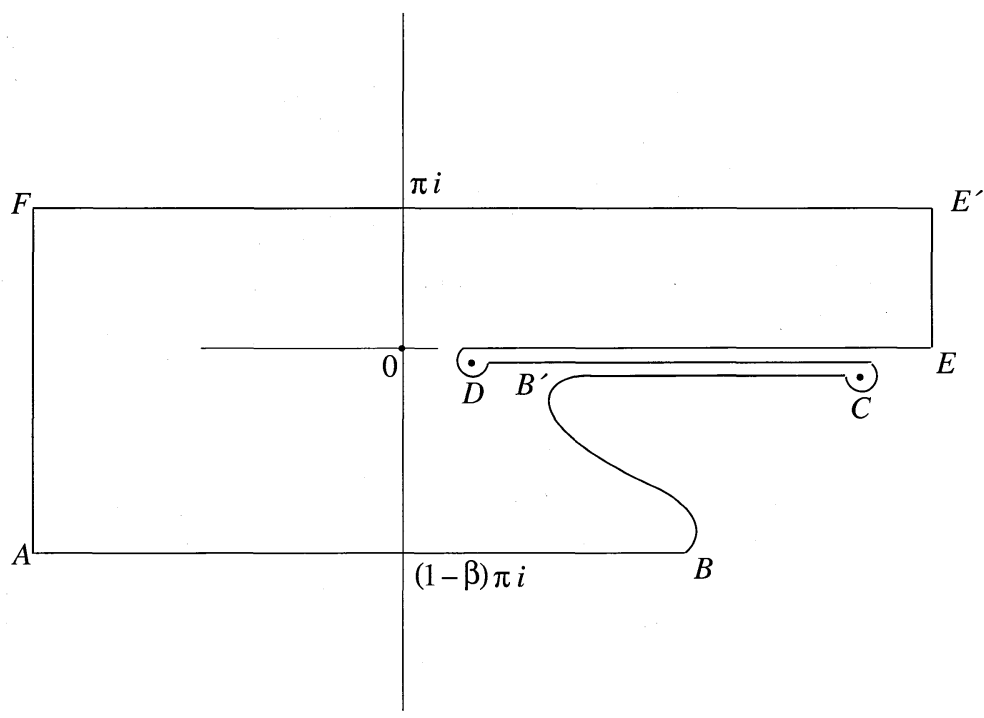

Figure 4. $Z$-plane $(\beta>1)$

We next consider the mapping $g: u \rightarrow Z$ defined by $g(u)=(1-\beta) \log u+u-$ $E / u+q$. By the same argument as above, when $u$ traverses once along the boundary of the region $A B B^{\prime} C D E E^{\prime} F A$ in Figure $5, Z$ goes once around the corresponding curve in the $Z$-plane. (Again, the lines in Figure 5 are only asymptotically straight.) Hence $g$ is one-to-one in the interior of the region $A B B^{\prime} C D E E^{\prime} F A$ in Figure 5. An approximation to the boundary curve $E^{\prime} F$ in Figure 5 is given by the equations

$$
(1-\beta) \arctan \frac{y}{x}+y=\pi \quad \text { if } x>0
$$

and

$$
(1-\beta)\left(\pi+\arctan \frac{y}{x}\right)+y=\pi \quad \text { if } x<0,
$$

where $u=x+i y$.

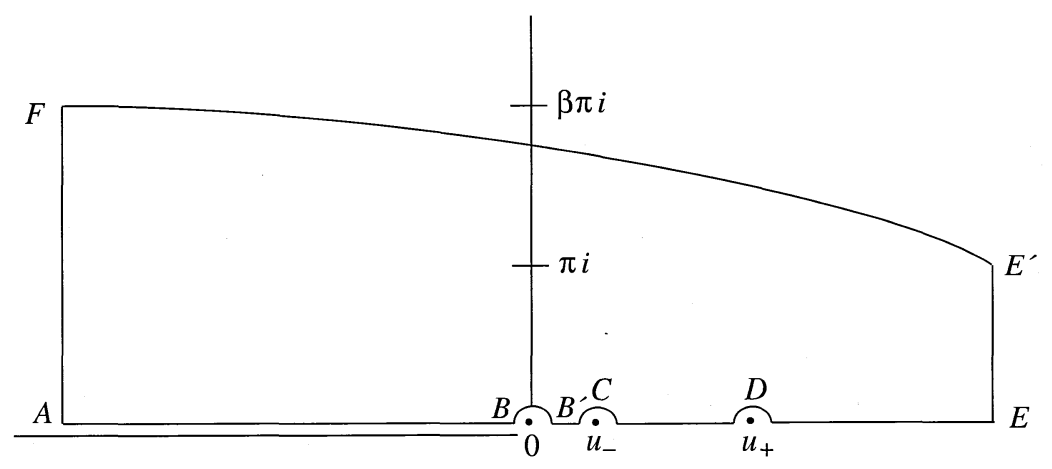

Figure 5. $u$-plane $(\beta>1)$

The transformation $s \leftrightarrow u$ is obtained by composing $f^{-1}: Z \rightarrow s$ and $g: u \rightarrow Z$. Since the transformations $s \leftrightarrow Z$ and $u \leftrightarrow Z$ are one-to-one within the boundary $A B B^{\prime} C D E E^{\prime} F A$, so is $s \leftrightarrow u$. Since the function $f(s)$ denoting the left-hand side of 
(3.1) satisfies $\overline{f(s)}=f(\bar{s})$, and since the function $g(u)$ denoting the right-hand side of (3.1) has the same property, the mapping of the lower half $s$-plane is deducible from Figures 3 and 5 by reflection with respect to the real axes. This establishes the one-to-one and analytic nature of the function $u(s, \beta)$ in the $s$-plane, except possibly at $s=s_{ \pm}$and on the two cuts from $s=1$ to $s=+\infty$ and from $s=0$ to $s=-\infty$. From the above argument (cf. Figures 3 and 5), it is also evident that neighborhoods of the points $s=s_{ \pm}$are mapped into neighborhoods of their corresponding images. Hence, $u(s, \beta)$ is bounded and analytic at these points. The same is true for the point $s=0$; see also the theorem in [11].

To emphasize what has been proved, we state again that the mapping $s \leftrightarrow u$, when $\beta>1$, is one-to-one and analytic from the cut $s$-plane to its image in the $u$-plane. In a similar manner, the same properties can be established when $0<\beta<1$. In the latter case, the regions bounded by $A B C D D^{\prime} E E^{\prime} F A$ in the $s^{-}, Z$-, and $u$-planes are shown in Figures $3^{\prime}, 4^{\prime}$, and $5^{\prime}$, respectively. Arguments similar to ours have been used previously by Copson [3, $\S 49]$, Olver [10, Chapter $9 ; \S 12.3]$, and Frenzen and Wong [5]. We have therefore proved (2.26) for the cases $0<\beta<1$ and $\beta>1$. The fact that this

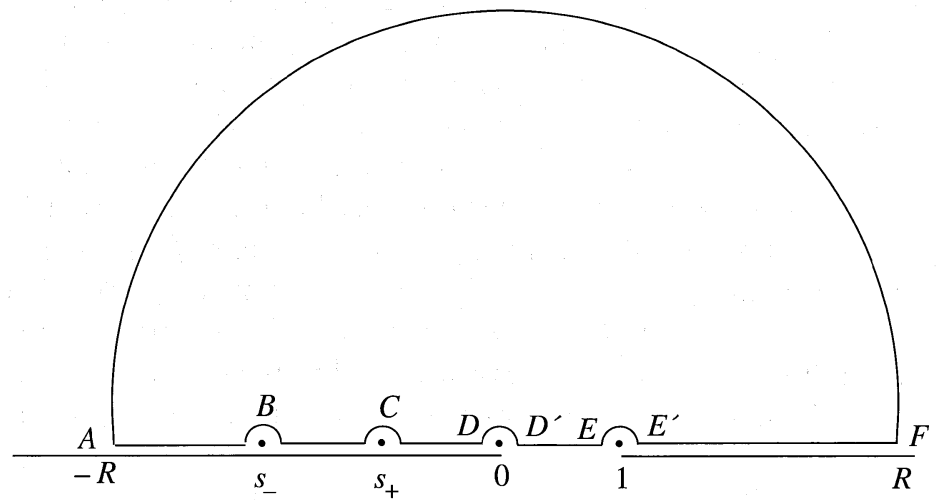

FiguRE $3^{\prime} . s$-plane $(0<\beta<1)$

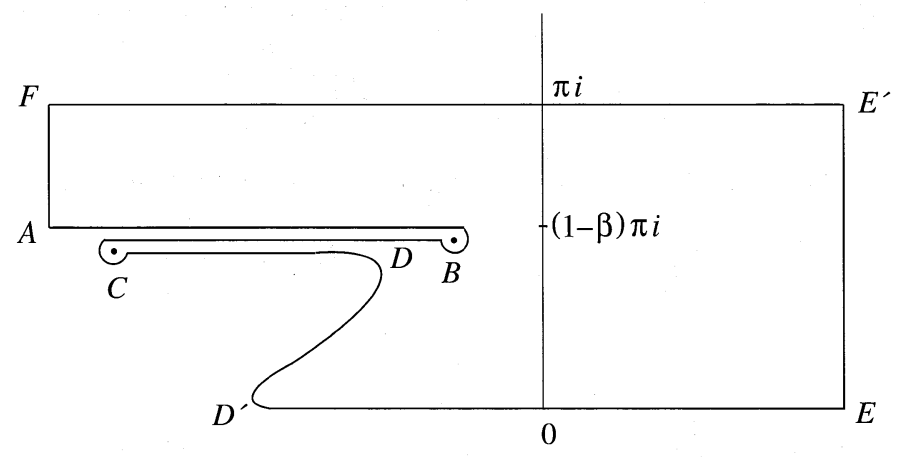

Figure $4^{\prime} . Z$-plane $(0<\beta<1)$

equation holds also for $\beta=1$ can either be demonstrated as in the other two cases or be using a continuity argument. To show that $u(s, \beta)$ is continuous in $\beta$, we first recall that it has already been proved in [11] that $u(s, \beta)$ is in fact analytic in both 


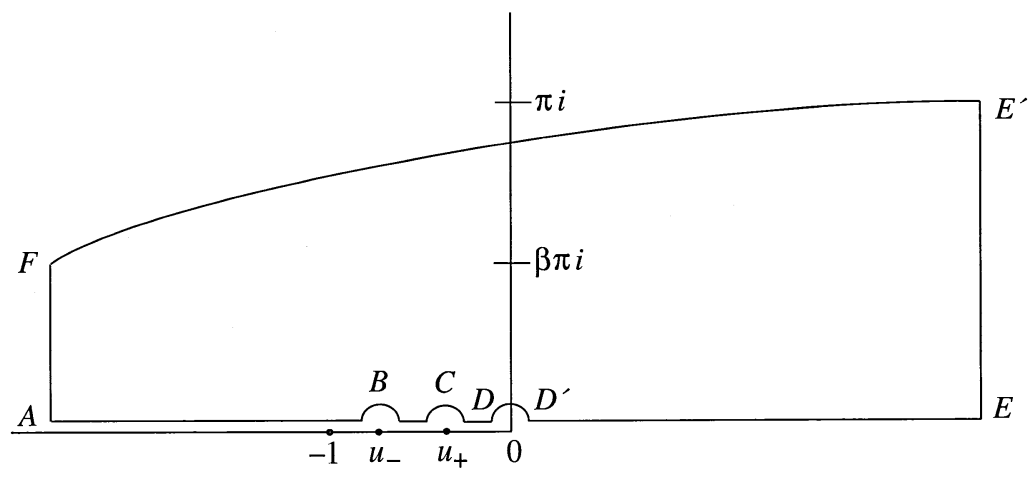

Figure $5^{\prime} . u$-plane $(0<\beta<1)$

variables in a neighborhood of $s=0$ and $\beta=1$. Thus we may assume $\beta \neq 1$. In this case, it is easily seen from (2.13) and $(2.14)$ that $p_{1}(\beta, n)$ and $p_{2}(\beta, n)$ are continuous in $\beta$. It is also clear from (2.20) and Figure 2 that $\alpha$ is a continuous function of $\beta$. Consequently, by (2.19) and (2.11), $E$ and $q$ are continuous in $\beta$. When $s=0$, $u$ is identically zero and hence is continuous in $\beta$. For fixed $s \neq 0,1$, the function $f(s, \beta, 1 / n)$ on the left-hand side of (2.6) is continuous in $\beta$. Now write this equation in the form $G(u, E, q, f)=0$, with $G$ being a differentiable function in each of its variables. As long as $\partial G / \partial u \neq 0$, by the Implicit Function Theorem there exists a function $u$ which is differentiable with respect to $E, q$, and $f$ and hence continuous in $\beta . \partial G / \partial u$ vanishes only at $u=u_{+}$and $u=u_{-}$, but from (2.8) it is evident that both $u_{+}$and $u_{-}$are continuous in $\beta$. (Recall that $u=u_{ \pm}$corresponds to $s=s_{ \pm}$.) It should be pointed out that the function $u$ actually depends also on the parameter $\gamma \equiv 1 / n$. However, its continuous dependence on $\gamma$ can be proved in a similar manner.

\section{Uniform asymptotic expansion}

We now return to the integral in (2.26). Put $h_{0}(u)=h(u)$ and write

$$
\begin{aligned}
h_{0}(u) & =a_{0}+\frac{1}{2} b_{0}\left(u-\frac{E}{u}\right)+\left[(1-\beta) \log u+u-\frac{E}{u}\right]^{\prime}\left[u g_{0}(u)\right] \\
& =a_{0}+\frac{1}{2} b_{0}\left(u-\frac{E}{u}\right)+\frac{\left(u-u_{+}\right)\left(u-u_{-}\right)}{u} g_{0}(u) .
\end{aligned}
$$

Since $u_{+} u_{-}=E$ (see $\left.\S 2\right)$, the coefficients $a_{0}$ and $b_{0}$ are given by

$$
a_{0}=\frac{h\left(u_{+}\right)+h\left(u_{-}\right)}{2}, \quad b_{0}=\frac{h\left(u_{+}\right)-h\left(u_{-}\right)}{u_{+}-u_{-}} .
$$

Thus

$$
\begin{aligned}
g_{0}(u)=\frac{u}{\left(u-u_{-}\right)\left(u-u_{+}\right)}\left\{h_{0}(u)\right. & -\frac{1}{2}\left[h_{0}\left(u_{+}\right)+h_{0}\left(u_{-}\right)\right] \\
& \left.-\frac{1}{2} \frac{h_{0}\left(u_{-}\right)-h_{0}\left(u_{+}\right)}{u_{-}-u_{+}}\left(u-\frac{E}{u}\right)\right\} .
\end{aligned}
$$

Clearly, $g_{0}(u)$ is analytic for $u \neq u_{ \pm}$and has removable singularities at $u=u_{ \pm}$since $E=u_{+} u_{-}$. From this we conclude that $g_{0}(u)$ is analytic everywhere in the domain 
of $h_{0}(u)$. Now substitute (4.1) into (2.26) and recall the integral representation [17, p. 176],

$$
J_{\nu}(z)=\frac{1}{2 \pi i} \int_{-\infty}^{(0+)} u^{-\nu-1} \exp \left\{\frac{1}{2} z\left(u-\frac{1}{u}\right)\right\} d u, \quad|\arg z|<\frac{\pi}{2}
$$

This leads to

$$
\frac{1}{n !} C_{n}^{(a)}(n \beta)=e^{a+n q} E^{-n(\beta-1) / 2}\left\{a_{0} J_{n(\beta-1)}(2 n \sqrt{E})+b_{0} \sqrt{E} J_{n(\beta-1)}^{\prime}(2 n \sqrt{E})+\varepsilon_{1}\right\},
$$

where

$\varepsilon_{1}=\frac{E^{n(\beta-1) / 2}}{2 \pi i} \int_{-\infty}^{(0+)}\left[(1-\beta) \log u+u-\frac{E}{u}\right]^{\prime} \exp \left\{n\left[(1-\beta) \log u+u-\frac{E}{u}\right]\right\} g_{0}(u) d u$.

To the last integral we apply an integration by parts. The integrated term vanishes, since $s(u) \sim-e^{-u / \beta}$ and $h_{0}(u)=O\left(u e^{u / \beta}\right)$ as $u \rightarrow-\infty$ for fixed $\beta>0$; see (2.6) and (2.7). The final result is

$$
\varepsilon_{1}=\frac{1}{n} \frac{E^{n(\beta-1) / 2}}{2 \pi i} \int_{-\infty}^{(0+)} u^{-n(\beta-1)-1} \exp \left\{n\left(u-\frac{E}{u}\right)\right\} h_{1}(u) d u
$$

where

$$
h_{1}(u)=-u g_{0}^{\prime}(u) \text {. }
$$

A similar technique of integration by parts has been used by Temme [13, eq. (5.13)].

The above procedure can be repeated. Thus, we define recursively

$$
\begin{gathered}
h_{0}(u)=h(u), \\
h_{k}(u)=a_{k}+\frac{1}{2} b_{k}\left(u-\frac{E}{u}\right)+\frac{\left(u-u_{-}\right)\left(u-u_{+}\right)}{u} g_{k}(u), \\
h_{k+1}(u)=-u g_{k}^{\prime}(u),
\end{gathered}
$$

As in (4.2), the coefficients $a_{k}$ and $b_{k}$ are given by

$$
a_{k}=\frac{h_{k}\left(u_{+}\right)+h_{k}\left(u_{-}\right)}{2}, \quad b_{k}=\frac{h_{k}\left(u_{+}\right)-h_{k}\left(u_{-}\right)}{u_{+}-u_{-}} .
$$

Using induction, we can show that $h_{k}(u)=O(1 / u)$ as $u \rightarrow-\infty$, for fixed $\beta$ and for all $k \geq 0$. From this it follows that we also have $g_{k}(u)=O(1)$, as $u \rightarrow-\infty$, for fixed $\beta$ and for all $k \geq 0$. Furthermore, we can show that for $k \geq 0, g_{k}(u)$ and $h_{k}(u)$ are analytic functions. Thus, for each $k$, both $a_{k}$ and $b_{k}$ are continuous in $\beta \in[\varepsilon, M]$, $0<\varepsilon<M<\infty$. Repeated application of integration by parts then gives

$$
\begin{aligned}
& \frac{1}{n !} C_{n}^{(a)}(n \beta)=e^{a+n q} E^{-n(\beta-1) / 2}\left\{J_{n(\beta-1)}(2 n \sqrt{E}) \sum_{k=0}^{p-1} a_{k} n^{-k}\right. \\
&\left.+\sqrt{E} J_{n(\beta-1)}^{\prime}(2 n \sqrt{E}) \sum_{k=0}^{p-1} b_{k} n^{-k}+\varepsilon_{p}\right\},
\end{aligned}
$$

where

$$
\varepsilon_{p}=\frac{1}{n^{p}} \frac{E^{n(\beta-1) / 2}}{2 \pi i} \int_{-\infty}^{(0+)} u^{-n(\beta-1)-1} \exp \left\{n\left(u-\frac{E}{u}\right)\right\} h_{p}(u) d u
$$


In order to show that (4.6) is a uniform asymptotic expansion for $0<\varepsilon \leq \beta \leq$ $M<\infty$, we prove that there exist positive numbers $M_{p}$ and $N_{p}$, independent of $\bar{\beta}$, such that

$$
\left|\varepsilon_{p}\right| \leq \frac{M_{p}}{n^{p}}\left|J_{n(\beta-1)}(2 n \sqrt{E})\right|+\frac{N_{p}}{n^{p}} \sqrt{E}\left|J_{n(\beta-1)}^{\prime}(2 n \sqrt{E})\right| .
$$

Since $a_{p}$ and $b_{p}$ are continuous in $\beta \in[\varepsilon, M]$, this estimate shows that the error term $\varepsilon_{p}$ has the same behavior as the first neglected term in the expansion (4.6).

The proof of (4.8) is divided into two separate cases: (i) $1 \leq \beta \leq M<\infty$ and (ii) $0<\varepsilon \leq \beta \leq 1$. Note that the argument $2 n \sqrt{E}$ of the Bessel function in (4.8) is large in both cases, and that the integral in (4.7) can be written in the form

$$
\varepsilon_{p}=\frac{1}{n^{p}} E^{n(\beta-1) / 2} \frac{1}{2 \pi i} \int_{-\infty}^{(0+)} e^{n[(1-\beta) \log u+u-E / u]} h_{p}(u) \frac{d u}{u} .
$$

Here it should be pointed out that the function $h_{p}(u)$ depends on the parameters $n$ and $\beta$; see the comments following (2.27). To estimate the integral in (4.9), we shall make use of the saddle point method, the stationary phase approximation and Laplace's approximation. Although $h_{p}(u)$ which is recursively defined through (4.4) is a complicated function, it is analytic in $u$ for all relevant values of $n$ and $\beta$. As a consequence, the dependence on $n$ and $\beta$ does not affect the final results; see [10, Chapter 9, Section 2]. We may therefore suppress this dependence in the following discussion.

We first consider case (i) and subdivide it into two subcases: (ia) $n(\beta-1)$ is unbounded and (ib) $n(\beta-1)$ is bounded. In subcase (ia), we observe that the function $\varphi(u)=(1-\beta) \log u+u-E / u$ has two saddle points located at $u=u_{ \pm}$. The steepest descent path through $u_{-}$is along the positive real axis, whereas the steepest descent path through $u_{+}$is tangent to a vertical line through $u_{+}$. Therefore, we deform the loop path of integration in (4.9) so that it passes through $u_{+}$; see Figure 6 . By the saddle point method [3, p. 93], we have

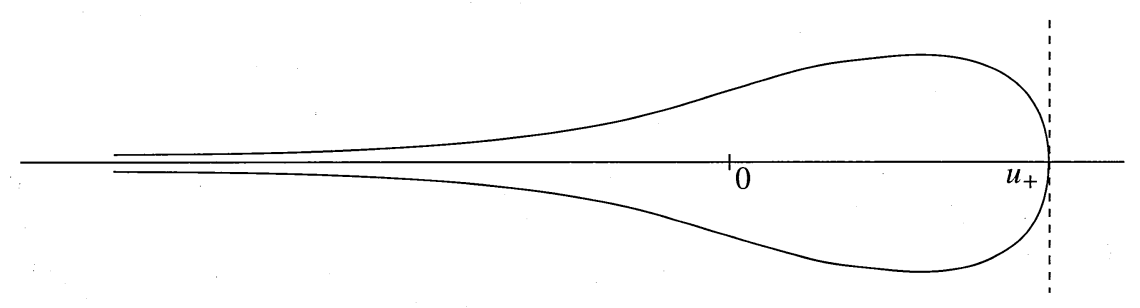

Figure 6

$$
\varepsilon_{p} \sim \frac{1}{n^{p}} E^{n(\beta-1) / 2} \frac{1}{2 \pi i} e^{n\left[(1-\beta) \log u_{+}+u_{+}-E / u_{+}\right]} \frac{h_{p}\left(u_{+}\right)}{u_{+}} \sqrt{\frac{-2 \pi}{n \varphi^{\prime \prime}\left(u_{+}\right)}}
$$

as $n \rightarrow \infty$. Since $u_{+} \sim \beta-1$ (cf. (2.8)), this reduces to

$$
\varepsilon_{p} \sim \frac{1}{n^{p}} E^{n(\beta-1) / 2}(\beta-1)^{n(1-\beta)} \exp \left\{n(\beta-1)-\frac{n E}{\beta-1}\right\} h_{p}\left(u_{+}\right) \frac{1}{\sqrt{2 \pi n(\beta-1)}} .
$$


From the uniform asymptotic expansion of $J_{\nu}(\nu z)$ and $J_{\nu}^{\prime}(\nu z)$, as $\nu \rightarrow \infty$, given in [1, pp. 368-369], it is easily verified that

$$
J_{n(\beta-1)}(2 n \sqrt{E}) \sim \frac{1}{\sqrt{2 \pi}} \frac{1}{(\beta-1)^{1 / 2}}\left(\frac{e \sqrt{E}}{\beta-1}\right)^{n(\beta-1)} e^{-n E /(\beta-1)} \frac{1}{\sqrt{n}}
$$

and

$$
J_{n(\beta-1)}^{\prime}(2 n \sqrt{E}) \sim \frac{1}{2}\left(\frac{\beta-1}{2 \pi E}\right)^{1 / 2}\left(\frac{e \sqrt{E}}{\beta-1}\right)^{n(\beta-1)} e^{-n E /(\beta-1)} \frac{1}{\sqrt{n}} .
$$

The estimate in (4.8) now follows from (4.11). In subcase (ib), we note that $n(\beta-1)$ is bounded, whereas $2 n \sqrt{E}$ approaches infinity. Hence

$$
u_{ \pm}=\frac{(\beta-1) \pm \sqrt{(\beta-1)^{2}-4 E}}{2}=\frac{\beta-1}{2} \pm \frac{i}{2} \sqrt{4 E-(\beta-1)^{2}} \approx \pm i \sqrt{E}
$$

as $n \rightarrow \infty$. This suggests that we deform the loop path of integration in (4.7) so that it consists of two straight lines along the negative real axis and a circle centered at the origin with radius $\sqrt{E}$. The circular portion of the integral is equal to

$$
\frac{1}{2 \pi n^{p}} \int_{-\pi}^{\pi} \exp \{i n[(1-\beta) \theta+2 \sqrt{E} \sin \theta]\} h_{p}\left(\sqrt{E} e^{i \theta}\right) d \theta .
$$

The critical points of the phase function in (4.15) are located at

$$
\theta_{ \pm}=\arccos \frac{\beta-1}{2 \sqrt{E}} \approx \pm \frac{\pi}{2}
$$

Hence, by the stationary phase approximation [19, p. 77], the expression in $(4.15)$ is asymptotic to

$$
\begin{aligned}
\frac{1}{2 n^{p}}\left(\frac{1}{\pi n \sqrt{E}}\right)^{1 / 2}\left\{h_{p}(\right. & i \sqrt{E}) e^{i n[(1-\beta) \pi / 2+2 \sqrt{E}-\pi / 4]} \\
& \left.+h_{p}(-i \sqrt{E}) e^{-i n[(1-\beta) \pi / 2+2 \sqrt{E}-\pi / 4]}\right\} .
\end{aligned}
$$

The portion of the integral in (4.7) along the two straight lines is given by

$$
\begin{aligned}
\frac{1}{n^{p}} \frac{E^{n(\beta-1) / 2}}{2 \pi i}\left\{e^{i \pi n(1-\beta)} \int_{\sqrt{E}}^{\infty} e^{-n[(\beta-1) \log r+r-E / r]} h_{p}\left(r e^{i \pi}\right) \frac{d r}{r}\right. \\
\left.-e^{-i \pi n(1-\beta)} \int_{\sqrt{E}}^{\infty} e^{-n[(\beta-1) \log r+r-E / r]} h_{p}\left(r e^{-i \pi}\right) \frac{d r}{r}\right\}
\end{aligned}
$$

The function $\psi(r)=(\beta-1) \log r+r-E / r$ is monotonically increasing in $\sqrt{E} \leq r<\infty$, and $\psi^{\prime}(\sqrt{E}) \neq 0$. Therefore, by Laplace's approximation [19, p. 57], the expression in (4.17) is bounded by

$$
\frac{1}{\pi n^{p}}\left(\frac{1}{n \sqrt{E}}\right)\left|h_{p}(-\sqrt{E})\right| \frac{1}{\psi^{\prime}(\sqrt{E})} .
$$

Note that $\psi^{\prime}(\sqrt{E}) \geq 2$ in this case. Comparing (4.16) and (4.18), we conclude that

$$
\varepsilon_{p}=E^{-1 / 4}\left|h_{p}(i \sqrt{E})\right| O\left(n^{-p-1 / 2}\right) .
$$


This is sufficient to justify (4.8), since $J_{\nu}(x)$ and $J_{\nu}^{\prime}(x)$ do not have common zeros, and since

$$
J_{n(\beta-1)}(2 n \sqrt{E}) \sim\left(\frac{1}{n \pi \sqrt{E}}\right)^{1 / 2} \cos \left\{2 n \sqrt{E}-n(\beta-1) \frac{\pi}{2}-\frac{\pi}{4}\right\}
$$

and

$$
\sqrt{E} J_{n(\beta-1)}^{\prime}(2 n \sqrt{E}) \sim-\left(\frac{\sqrt{E}}{n \pi}\right)^{1 / 2} \sin \left\{2 n \sqrt{E}-n(\beta-1) \frac{\pi}{2}-\frac{\pi}{4}\right\}
$$

as $n \rightarrow \infty$, when $n(\beta-1)$ is a bounded quantity.

We next consider case (ii) and again divide it into two subcases: (iia) $n(\beta-1) \rightarrow-\infty$ and (iib) $n(\beta-1)$ is bounded. Since $E$ is positive, the argument for subcase (iib) is similar to that of case (ib) and hence will be omitted. In subcase (iia), we note that $u_{-}<u_{+}<0, u_{-} \sim \beta-1$ and $u_{+} \sim 0$; see Figure $5^{\prime}$. It can be shown that the steepest descent path through $u_{-}$is along the negative real axis and that the steepest descent path through $u_{+}$is tangent to a vertical line through $u_{+}$. Therefore, we deform the loop path of integration in (4.9) so that it passes through $u_{-}$on both edges of the cut; see Figure 7. By the saddle point method, we have

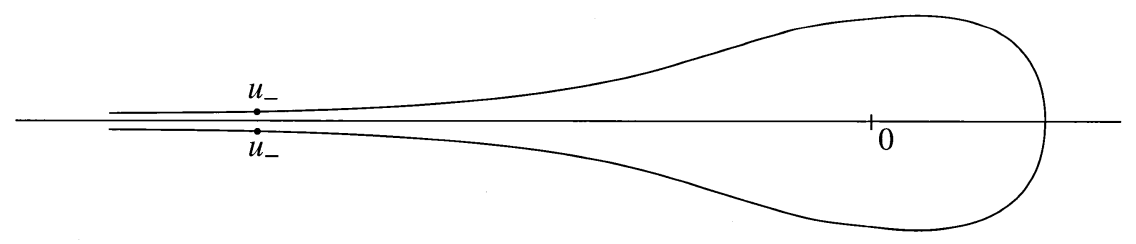

Figure 7

$$
\begin{gathered}
\varepsilon_{p} \sim \frac{1}{n^{p}} E^{n(\beta-1) / 2}(1-\beta)^{n(1-\beta)-1 / 2} \exp \left\{n(\beta-1)-\frac{n E}{\beta-1}\right\} \\
\times \sin [\pi n(1-\beta)] h_{p}(\beta-1) \sqrt{\frac{2}{\pi n}}
\end{gathered}
$$

as $n \rightarrow \infty$. Here we have made use of the fact that $u_{-} \sim \beta-1$. To show that (4.20) implies (4.8), we note that

$$
\begin{aligned}
J_{n(\beta-1)} & (2 n \sqrt{E}) \\
& \sim \sqrt{\frac{2}{\pi n(1-\beta)}} \sin [n(1-\beta) \pi]\left(\frac{1-\beta}{e \sqrt{E}}\right)^{n(1-\beta)} e^{n E /(1-\beta)}
\end{aligned}
$$

and

$$
\begin{aligned}
& J_{n(\beta-1)}^{\prime}(2 n \sqrt{E}) \\
& \quad \sim-\sqrt{\frac{1}{2 \pi n(1-\beta) E}} \sin [n(1-\beta) \pi]\left(\frac{1-\beta}{e \sqrt{E}}\right)^{n(1-\beta)} e^{n E /(1-\beta)},
\end{aligned}
$$

when $n \rightarrow \infty$ and $\beta<1$ (i.e., $n(\beta-1) \rightarrow-\infty)$. Asymptotic formulas (4.21) and (4.22) can be obtained from the uniform asymptotic expansions of $H_{\nu}^{(1)}(\nu z)$ and $H_{\nu}^{(2)}(\nu z)$ as 
$\nu \rightarrow \infty$ given in [1, pp. 368-369], and by using the connection formula

$$
J_{-\nu}(z)=\frac{1}{2}\left[e^{\nu \pi i} H_{\nu}^{(1)}(z)+e^{-\nu \pi i} H_{\nu}^{(2)}(z)\right]
$$

see [10, p. 239]. This completes the proof of (4.8).

The leading coefficients $a_{0}$ and $b_{0}$ in (4.6) can be calculated as follows. From (2.27) we have

$$
h\left(u_{-}\right)=\left.\frac{u_{-}}{\left(1-s_{-}\right) s_{-}} \frac{d s}{d u}\right|_{u=u_{-}}, \quad h\left(u_{+}\right)=\left.\frac{u_{+}}{\left(1-s_{+}\right) s_{+}} \frac{d s}{d u}\right|_{u=u_{+}} .
$$

Since $u=u_{ \pm}$correspond to $s=s_{ \pm}$, differentiating (2.7) with respect to $u$ gives

$$
\left.\left(\frac{d s}{d u}\right)^{2}\right|_{u=u_{ \pm}}=\frac{\frac{\beta-1}{u_{ \pm}^{2}}-\frac{2 E}{u_{ \pm}^{3}}}{\frac{\beta-1}{s_{ \pm}^{2}}+\frac{1}{\left(1-s_{ \pm}\right)^{2}}-\frac{2(a / n)}{s_{ \pm}^{3}}} .
$$

To determine the sign of the square root of the right-hand side of (4.25) for $d s / d u$ at $u=u_{ \pm}$, we note from the second equality in (2.27) that $h\left(u_{ \pm}\right)$is positive when $u_{ \pm}$ are real. Hence we must take the positive square root. The values of $a_{0}$ and $b_{0}$ are now easily obtained from (4.2).

\section{Local behavior}

Taking the dominant term of the expansion in (4.6), we have

$$
\begin{aligned}
\frac{1}{n !} C_{n}^{(a)}(n \beta) \sim e^{a+n q} E^{-n(\beta-1) / 2}\left\{a_{0}\right. & J_{n(\beta-1)}(2 n \sqrt{E}) \\
& \left.+b_{0} \sqrt{E} J_{n(\beta-1)}^{\prime}(2 n \sqrt{E})\right\},
\end{aligned}
$$

as $n \rightarrow \infty$, holding uniformly for $0<\varepsilon \leq \beta \leq M<\infty$. If $x=n \beta$ is restricted to one of the seven regions encountered in the introduction, then (5.1) should reduce to one of the asymptotic formulas given by Goh [6]. Here we shall consider only the four regions: (1) $1+\varepsilon \leq \beta \leq M<\infty$, (3) $\beta=\beta_{+}+t / n^{5 / 6}$, (5) $\beta=\beta_{-}+t / n^{5 / 6}, t$ real, and (7) $0<\varepsilon \leq \beta \leq 1-\varepsilon$. Arguments for the other three regions are similar.

For $\beta \geq 1+\varepsilon$, we have from (2.4) and (2.8)

$$
s_{+} \sim \frac{\beta-1}{\beta}, \quad s_{-} \sim \frac{a / n}{\beta-1}, \quad u_{+} \sim \beta-1, \quad u_{-} \sim \frac{E}{\beta-1} .
$$

Hence, by (4.24) and (4.25), $h\left(u_{+}\right) \sim \sqrt{\beta}$ and $h\left(u_{-}\right) \sim 1$. Consequently,

$$
a_{0} \sim \frac{1}{2}(\sqrt{\beta}+1), \quad b_{0} \sim \frac{1}{\sqrt{\beta}+1} .
$$

Inserting (2.17), (4.12), and (4.13) into (5.1) gives

$$
\frac{1}{n !} C_{n}^{(a)}(n \beta) \sim\left(\frac{\beta}{\beta-1}\right)^{1 / 2} e^{a /(1-\beta)} \beta^{n \beta}(\beta-1)^{n(1-\beta)} \frac{1}{\sqrt{2 \pi n}},
$$

which agrees with equation (5) in [6].

For $0<\beta \leq 1-\varepsilon$, we have

$$
s_{+} \sim \frac{a / n}{\beta-1}, \quad s_{-} \sim \frac{\beta-1}{\beta}, \quad u_{+} \sim \frac{E}{\beta-1}, \quad u_{-} \sim \beta-1 .
$$


Hence, $h\left(u_{+}\right) \sim 1$ and $h\left(u_{-}\right) \sim \sqrt{\beta}$. Therefore, (5.2) again holds. Applying (2.17), (4.21), and (4.22) to (5.1), we obtain

$$
\frac{1}{n !} C_{n}^{(a)}(n \beta) \sim\left(\frac{\beta}{1-\beta}\right)^{1 / 2} e^{a /(1-\beta)} \beta^{n \beta}(1-\beta)^{n(1-\beta)} \sin [n(1-\beta) \pi] \sqrt{\frac{2}{\pi n}},
$$

which is equation (84) in [6].

In the case when $\beta$ is near $\beta_{+}$, the computation is more complicated. Here we have

$$
\beta=\beta_{+}+\frac{t}{n^{5 / 6}}=1+2 \sqrt{\frac{a}{n}}+\frac{t}{n^{5 / 6}}+\frac{a}{n},
$$

$t$ being a bounded real number. From (2.13) and (2.14) it follows that

$$
\begin{aligned}
p_{1}(\beta, n) \sim 2 & \sqrt{\frac{a}{n}} \log n-2 \sqrt{\frac{a}{n}} \log a+\frac{t}{n^{5 / 6}} \log n \\
& -\frac{t}{n^{5 / 6}} \log a+\frac{a}{n}\left(1+\log \frac{n}{a}\right)
\end{aligned}
$$

and

$$
p_{2}(\beta, n)=-\frac{4}{3} \frac{t^{3 / 2}}{a^{1 / 4}} \frac{1}{n}+o\left(\frac{1}{n^{7 / 6}}\right) .
$$

(In the derivation of the last asymptotic equality, we have actually considered two separate cases: $t \geq 0$ and $t<0$.) As in the proof of the lemma in $\S 2$, we now solve $E$ in (2.12) asymptotically for $t \geq 0$. First, substituting (5.3) and (5.5) into (2.12), we readily see that $E \sim C_{0} a / n$, where $C_{0}$ is a constant $\leq 1$. Next, we set $E=C_{0} a n^{-1}+\rho$ where $\rho=o\left(n^{-1}\right)$, and again substitute it into (2.12). This leads to the results $C_{0}=1$ and $\rho=O\left(n^{-4 / 3}\right)$. Now, we write

$$
E=\frac{a}{n}+C_{1} \frac{t \sqrt{a}}{n^{4 / 3}}+\varepsilon
$$

where $\varepsilon=o\left(n^{-4 / 3}\right)$. Substituting this expression into (2.12) yields $C_{1}=0$ and $\varepsilon=O\left(n^{-3 / 2}\right)$. Continuing in this manner, we arrive at the final result

$$
E=\frac{a}{n}+\left(\frac{a}{n}\right)^{3 / 2}+o\left(\frac{1}{n^{3 / 2}}\right)
$$

A combination of (2.11), (5.4), and (5.6) also gives

$$
q \sim \frac{3}{2} \frac{a}{n}
$$

It can be shown that the same results hold for $t<0$. It is interesting to note that both (5.6) and (5.7) could have been derived formally from the lemma in $\S 2$, although the results there were proved only for fixed $\beta$. To obtain the behavior of $J_{n(\beta-1)}(2 n \sqrt{E})$ and $J_{n(\beta-1)}^{\prime}(2 n \sqrt{E})$ in this case, we use the uniform asymptotic approximations of $J_{\nu}(\nu z)$ and $J_{\nu}^{\prime}(\nu z)$ given in [1, pp. 368-369]. With $\nu=n(\beta-1)=2 \sqrt{a n}+t n^{1 / 6}+a$ and

we have

$$
z=\frac{2 n \sqrt{E}}{n(\beta-1)}=\frac{2 \sqrt{a n}+a+o(1)}{2 \sqrt{a n}+t n^{1 / 6}+a}=1-\frac{t}{2 \sqrt{a}} \frac{1}{n^{1 / 3}}+o\left(\frac{1}{n^{1 / 2}}\right),
$$

$$
J_{n(\beta-1)}(2 n \sqrt{E}) \sim(a n)^{-\frac{1}{6}} \mathrm{Ai}\left(t a^{-\frac{1}{6}}\right)
$$


and

$$
J_{n(\beta-1)}^{\prime}(2 n \sqrt{E}) \sim-(a n)^{-\frac{1}{3}} \operatorname{Ai}^{\prime}\left(t a^{-\frac{1}{6}}\right) .
$$

By (5.6),

$$
E^{-n(\beta-1) / 2} \sim e^{-a}\left(\frac{n}{a}\right)^{\sqrt{a n}+(t / 2) n^{1 / 6}+a / 2} .
$$

Inserting (5.7), (5.8), (5.9), and (5.10) into (5.1) gives

$$
\frac{1}{n !} C_{n}^{(a)}(n \beta) \sim e^{3 a / 2}\left(\frac{n}{a}\right)^{\sqrt{a n}+(t / 2) n^{1 / 6}+a / 2}(a n)^{-1 / 6} \operatorname{Ai}\left(t a^{-1 / 6}\right),
$$

since $a_{0} \sim 1$ and (5.9) is of lower asymptotic order of magnitude than (5.8). Here our result again agrees with that of Goh [6, eq. (30)].

In region (5), we have

$$
\beta=\beta_{-}+\frac{t}{n^{5 / 6}}=1-2 \sqrt{\frac{a}{n}}+\frac{t}{n^{5 / 6}}+\frac{a}{n} .
$$

Straightforward calculation from (2.13) and (2.14) gives

$$
p_{1}(\beta, n) \sim\left(2 \sqrt{\frac{a}{n}}-\frac{t}{n^{5 / 6}}-\frac{a}{n}\right) \log \left(\frac{a}{n}\right)+\frac{a}{n}
$$

and

$$
p_{2}(\beta, n)=-\frac{4}{3} \frac{(-t)^{3 / 2}}{a^{1 / 4}} \frac{1}{n}+o\left(\frac{1}{n^{7 / 6}}\right),
$$

where $(-t)^{3 / 2}$ is real and positive when $t<0$. By the perturbation technique used before, we obtain

$$
E=\frac{a}{n}-\left(\frac{a}{n}\right)^{3 / 2}+o\left(\frac{1}{n^{3 / 2}}\right)
$$

This result can again be derived formally from $(2.15)$. Note that $n(\beta-1)$ is negative in the present case. Hence, to obtain the behavior of $J_{n(\beta-1)}(2 n \sqrt{E})$ and $J_{n(\beta-1)}^{\prime}(2 n \sqrt{E})$, we again appeal to (4.23) and the uniform asymptotic expansions of $H_{\nu}^{(1)}(\nu z)$ and $H_{\nu}^{(2)}(\nu z)$ given in [1, pp. 368-369]. With $\nu=-n(\beta-1)=2 \sqrt{a n}-t n^{1 / 6}-a$ and

$$
z=-\frac{2 \sqrt{E}}{\beta-1} \sim 1+\frac{t}{2 \sqrt{a}} \frac{1}{n^{1 / 3}},
$$

the results are

$$
\begin{array}{r}
J_{n(\beta-1)}(2 n \sqrt{E}) \sim(a n)^{-1 / 6}\left[e^{(\nu \pi-\pi / 3) i} \operatorname{Ai}\left(e^{-\pi i / 3} t a^{-1 / 6}\right)\right. \\
\left.+e^{-(\nu \pi-\pi / 3) i} \operatorname{Ai}\left(e^{\pi i / 3} t a^{-1 / 6}\right)\right]
\end{array}
$$

and

$$
\begin{aligned}
J_{n(\beta-1)}^{\prime}(2 n \sqrt{E}) \sim 2(a n)^{-1 / 3}\left[e^{(\nu \pi+4 \pi / 3) i} \operatorname{Ai}^{\prime}\left(e^{-\pi i / 3} t a^{-1 / 6}\right)\right. \\
\left.+e^{-(\nu \pi+4 \pi / 3) i} \operatorname{Ai}^{\prime}\left(e^{\pi i / 3} t a^{-1 / 6}\right)\right] .
\end{aligned}
$$


Clearly, $\sqrt{E} J_{n(\beta-1)}^{\prime}(2 n \sqrt{E})$ is of lower asymptotic order of magnitude than is $J_{n(\beta-1)}(2 n \sqrt{E})$. Since $a_{0} \sim 1$ and $b_{0} \sim \frac{1}{2}$, the second term in (5.1) can be neglected. From (2.11), it is easily calculated that

$$
q \sim \frac{3}{2} \frac{a}{n}
$$

Therefore, (5.1) gives

$$
\begin{aligned}
& \frac{1}{n !} C_{n}^{(a)}(n \beta) \sim e^{3 a / 2}\left(\frac{n}{a}\right)^{\left(-2 \sqrt{a n}+t n^{1 / 6}+a\right) / 2}(a n)^{-1 / 6} \\
& \times 2 \operatorname{Re}\left[e^{-\left(2 \sqrt{a n}-t n^{1 / 6}-a-1 / 3\right) \pi i} \operatorname{Ai}\left(e^{\pi i / 3} t a^{-1 / 6}\right)\right],
\end{aligned}
$$

or equivalently

$$
\begin{aligned}
\frac{1}{n !} C_{n}^{(a)}(x) \sim e^{3 a / 2}\left(\frac{n}{a}\right)^{(x-n) / 2}(a n)^{-1 / 6}(-1)^{n} \\
\times 2 \operatorname{Re}\left[e^{(x \pi+\pi / 3) i} \operatorname{Ai}\left(e^{\pi i / 3} t a^{-1 / 6}\right)\right],
\end{aligned}
$$

where $x=n \beta=n-2 \sqrt{a n}+t n^{1 / 6}+a$. As in [6], this result can also be expressed in terms of Scorer's function

$$
\operatorname{Hi}(z)=\frac{1}{\pi} \int_{0}^{\infty} e^{-1 / 3 t^{3}+z t} d t
$$

To do this, we make use of the facts

$$
\left[e^{-\pi i / 6} \operatorname{Hi}\left(z e^{\pi i / 3}\right)+e^{\pi i / 6} \operatorname{Hi}\left(z e^{-\pi i / 3}\right)\right]=2 \operatorname{Ai}(-z)
$$

and

$$
\operatorname{Ai}(z)+e^{2 \pi i / 3} \operatorname{Ai}\left(z e^{2 \pi i / 3}\right)+e^{-2 \pi i / 3} \operatorname{Ai}\left(z e^{-2 \pi i / 3}\right)=0
$$

see $[10$ p. 332 and p. 55]. The final result is

$$
\begin{aligned}
\frac{1}{n !} C_{n}^{(a)}(x) & \sim e^{3 a / 2}\left(\frac{n}{a}\right)^{(x-n) / 2}(a n)^{-1 / 6}(-1)^{n} \\
& \times\left\{\operatorname{Re}\left[e^{(x \pi+\pi / 6) i} \operatorname{Hi}\left(t a^{-1 / 6} e^{-\pi i / 3}\right)\right]-\sin \pi x \operatorname{Hi}\left(-t a^{-1 / 6}\right)\right\},
\end{aligned}
$$

which is equation (51) in [6].

Acknowledgement. We would like to express our sincere thanks to Professor W. M. Y. Goh for allowing us to make reference to his paper [6] prior to its publication. We are also grateful to the referees for their careful reading of the manuscript and several very helpful suggestions.

\section{References}

1. M. Abramowitz and I. A. Stegun, eds., Handbook of Mathematical Functions, National Bureau of Standards, Appl. Math. Ser. 55, Washington, D.C., 1964.

2. T. S. Chihara, An Introduction to Orthogonal Polynomials, Gordon and Breach, New York, 1978.

3. E. T. Copson, Asymptotic Expansions, Cambridge University Press, Cambridge, 1965.

4. A. Erdélyi, Asymptotic forms for Laguerre polynomials, J. Indian Math. Soc., Golden Jubilee Commemoration Volume 24 (1960), 235-250.

5. C. L. Frenzen and R. Wong, Uniform asymptotic expansions of Laguerre polynomials, SIAM J. Math. Anal. 19 (1988), 1232-1248.

6. W. M. Y. Goh, Plancherel-Rotach asymptotics for the Charlier polynomials, Constr. Approx., to appear. 
7. M. Maejima and W. Van Assche, Probabilistic proofs of asymptotic formulas for some classical polynomials, Math. Proc. Cambridge Philos. Soc. 97 (1985), 499-510.

8. A. I. Markushevich, Theory of Functions of a Complex Variable, 2nd ed., Chelsea, New York, 1977.

9. A. Olde Daalhuis and N. M. Temme, Uniform Airy type expansions of integrals, SIAM J. Math. Anal., to appear.

10. F. W. J. Olver, Asymptotics and Special Functions, Academic Press, New York, 1974.

11. C. K. Qu and R. Wong, Transformation to canonical form for uniform asymptotic expansions, J. Math. Anal. Appl. 149 (1990), 210-219.

12. G. Szegö, Orthogonal Polynomials, 4th ed., Colloquium Publications, Vol. 23, Amer. Math. Soc., Providence, RI, 1975.

13. N. M. Temme, Laguerre polynomials: asymptotics for large degree, CWI Report AM-R8610.

14. E. C. Titchmarsch, Theory of Functions, 2nd ed., Oxford University Press, Oxford, 1939.

15. F. G. Tricomi, Funzioni Ipergeometriche Confluenti, Edizione Cremonese, Roma, 1954.

16. F. Ursell, Integrals with a large parameter: Paths and descent and conformal mapping, Math. Proc. Cambridge Philos. Soc. 67 (1970), 371-381.

17. G. N. Watson, A Treatise on the Theory of Bessel Functions, 2nd ed., Cambridge University Press, London, 1944.

18. J. Wimp and D. Zeilberger, Resurrecting the asymptotics of linear recurrences, J. Math. Anal. Appl. 111 (1985), 165-176.

19. R. Wong, Asymptotic Approximations of Integrals, Academic Press, New York, 1989.

20. R. Wong and H. Li, Asymptotic expansions for second-order linear difference equations, J. Comput. Appl. Math. 41 (1992), 65-94.

Department of Basic Science, (Mathematics), China Textile University, Shanghai, China

Department of Applied Mathematics, University of Manitoba, Winnipeg, Manitoba, Canada R3T $2 \mathrm{~N} 2$ 